\title{
Gestión de riesgos en la nueva normalidad post Covid-19
}

\author{
Risk management in the new post-covid-19 normality \\ Remo Eusebio Zacarías Rodríguez ${ }^{1, a}$, Victoriano Eusebio Zacarías Rodríguez ${ }^{1, b}$, Sara Ricardina Zacarías \\ Vallejos ${ }^{1, c}$
}

\section{RESUMEN}

La investigación Gestión de riesgos en la nueva normalidad post covid-19, el retorno a las actividades tanto profesionales, educativas y cotidianas no será igual que antes. Los ciudadanos estarán preocupados por su bioseguridad, por los riesgos a los que estarán expuestos en los ambientes físicos y el comportamiento de los demás ciudadanos. En este escenario, el principal desafío como organizaciones, será traducir en hechos concretos y prácticos. El objetivo del presente trabajo fue determinar la influencia de la gestión de riesgos en la nueva normalidad post COVID 19. El trabajo de investigación es básico, diseño no experimental, de corte transversal correlacionalcausal, la muestra 107 empresarios socios de la Cámara de Comercio de Huancayo. Se encontró que la gestión de riegos emergentes como la prevención, documentación y la gestión cuentan con un 37\%. En las actividades de seguimiento, control y análisis de riesgos con un 35\% de aplicación., en cuanto a la nueva normalidad post covid-19, se cuentan con un 39\% el plan para la vigilancia, prevención y control de Covid-19 en el trabajo. Con un $39 \%$ cuentan con equipos de protección personal anti Covid-19 y protocolos para la atención y los procesos en el trabajo. Podemos concluir que a un nivel de significancia del 0,05 la gestión de riesgos influye significativamente en la nueva normalidad post COVID 19, lo cual significa que la gestión de riesgos influye hasta un 58.5\% en la nueva normalidad post COVID 19.

PALABRAS CLAVE: Gestión de Riesgos, nueva normalidad post covid-19.

\section{SUMMARY}

The investigation Risk management in the new post-covid-19 normality, the return to professional, educational and daily activities will not be the same as before. Citizens will be concerned about their biosecurity, about the risks to which they will be exposed in physical environments and the behavior of other citizens. In this scenario, the main challenge as organizations will be to translate into concrete and practical facts. The objective was to determine the influence of risk management in the new normality post COVID 19. Material and Method: the research work is basic, non-experimental design, of correlational-causal cross-section, the sample 107 business partners of the Chamber of Commerce of Huancayo. The management of emerging risks such as prevention, documentation and management have 37\%. In the monitoring, control and risk analysis activities with 35\% of application, as for the new post-covid-19 normality, there is a 39\% plan for the surveillance, prevention and control of Covid-19 in the job.

\footnotetext{
Universidad Privada de Huancayo Franklin Roosevelt. Huancayo, Perú.

Docente; Lic. en Administración; Magister. ORCID ID: 0000-0002-7648-9274

Docente; Lic. en Administración; Magister. ORCID ID: 0000-0002-5842-555X

Docente; Lic. en Administración; Magister. ORCID ID: 0000-0003-3481-198X
} 
With 39\% they have personal protection equipment against Covid-19 and protocols for care and processes at work. We found a significance level of 0.05 , risk management significantly influences the new post-COVID 19 normal, which means that risk management influences up to $58.5 \%$ of the new post-COVID 19 normal.

KEYWORDS: Risk Management, the new normality post covid-19.

\section{INTRODUCCIÓN}

Cuando se inició la crisis sanitaria, se vislumbró tres probables Escenarios de recuperación para las organizaciones, considerando diversos factores; sin embargo, hemos observado que el desarrollo de la pandemia ha sido más lento de lo esperado, y la recuperación según los especialistas podría retrasarse hasta 2022. Entonces nos preguntamos; ¿Cómo enfrentar la incertidumbre de la nueva normalidad?, para ello se debe adoptar una nueva filosofía de trabajo, haciendo las cosas de manera diferente a la acostumbrada, convenciéndonos de no gestionar igual que antes y evitando aplicar las fórmulas que conocíamos, con el objetivo de hacer sostenible y próspero nuestro futuro y el de la sociedad.

Según Pérez (2021), el panorama no es tan simple ya que a pesar de que es posible que logremos establecer estrategias que generen armonía entre nuestros equipos dentro de los grandes sectores, también existirán decisiones que provocarán puntos de vista encontrados, como el caso de, determinar si se debe anteponer el aspecto sanitario o el de negocios, o bien, qué procesos de respuesta a la pandemia se mantendrán y qué otros se modificarán o sustituirán. Para ello se debe realizar un diagnóstico previo, reconociendo si las empresas están preparadas para recibir a los empleados y/o terceros en sus oficinas, y teniendo conocimiento de todos los riesgos e implicaciones operativas, se podrán llevar a cabo acciones e inversiones que realmente faciliten la pronta recuperación de la organización.

Se dijo que el trabajo remoto llegó para quedarse y que será una parte fundamental del correcto desarrollo de las operaciones de la empresa, así como de la manera de interactuar de su gente. Sin embargo, esta modalidad de trabajo requerirá destrezas de liderazgo más desafiantes, e implicará atender diversos retos de infraestructura y de comunicaciones, definir de manera diferenciada roles y funciones, así como diseñar un esquema inteligente de medición del desempeño organizacional. Pero muchas organizaciones tanto públicas y privadas están haciendo un híbrido entre el trabajo remoto y presencial tal como se puede apreciar en nuestra ciudad, pero hace necesario en ellos los riegos a los que están expuestos y la propuesta es como gestionar esos riesgos. Otro aspecto que está exigiendo un esfuerzo significativo para las empresas es la necesidad de garantizar un entorno de trabajo seguro y confiable. La credibilidad de una organización ante la sociedad, y en especial ante colaboradores y clientes, en un entorno posterior al COVID 19, demandará la creación de confianza a todos los niveles. Aspectos cotidianos y básico como ir a la cafetería, atender clientes o proveedores, y usar los servicios sanitarios, las salas de reunión o incluso las fotocopiadoras requerirá no solo de la construcción de una cultura de salud ocupacional, sino también de conocer muy bien las formas de prevenir el contagio del virus en los entornos de trabajo, lo cual podría implicar la modificación de horarios y de las condiciones de uso de espacios, entre muchos otros aspectos.

Además, deberá considerarse, en el nuevo Business As Usual (lo de siempre), la planificación estratégica y financiera con base en escenarios a corto y mediano plazos, y el diseño de acciones e iniciativas, dependiendo de cómo evolucione la pandemia. Hoy, una buena parte de los planes estratégicos que estaba en uso por las organizaciones ha experimentado cambios profundos en sus premisas fundamentales y elementos muy diferentes a considerar en la priorización de iniciativas y metas, la que se hace necesario actualizar e incluir a esta pandemia.

Por otro lado, el uso científico del análisis de datos con múltiples propósitos organizacionales será cada vez más relevante. Esto implica que las herramientas de inteligencia de negocios, el modelamiento de productos y servicios, la definición de campañas comerciales y la interacción con los mercados deberán contemplar las nuevas tendencias de comportamiento del público objetivo y de los mismos colaboradores de la empresa. Paralelamente, a través de la gestión científica de los datos de la organización, se tendrá que construir y mantener un sistema permanente de reportes, tanto de la gestión como del desempeño organizacional entiempo real, el cual permita identificar riesgos y amenazas de forma oportuna. 
Por último, se necesitará acelerar el esfuerzo digital, mediante los componentes clave de la cadena de suministro (abastecimiento): la entrega de servicios y productos a través de plataformas; programas y recursos cada día más automatizados; mayor uso de tecnologías como el blockchain, RPA (robotic process automation), aprendizaje mecanizado; y lenguajes cognitivos y predictivos que permitan atender de forma ágil y segura las necesidades y demandas de clientes y mercados, procurando la fidelidad e incremento de la base de clientes y optimizando el uso de recursos. La pandemia ha demostrado que aquellas organizaciones que iniciaron su transformación digital resolvieron más rápido los desafíos del presente, en comparación con aquellas que no se preocuparon a tiempo por estos temas y que hoy enfrentan grandes retos para mantener y atender a sus clientes, así como para encontrar el equilibrio en su eficiencia operacional.

Frente a lo mencionado anteriormente planteamos el siguiente problema general: ¿Cómo influye la gestión de riesgos en la nueva normalidad post COVID 19? y como problemas específicos; ¿Cómo influye la visión holística en la nueva normalidad post COVID 19?, ¿Cómo influye la gestión de riesgos emergentes en la nueva normalidad post COVID 19? y ¿Cómo influye la gestión de eventos significativos en la nueva normalidad post COVID 19?

La investigación se justifica ya que a nivel país, existe la preocupación de los empresarios a volver a la nueva normalidad a prestar sus servicios empresariales, para ofertar sus productos $\mathrm{y} / \mathrm{o}$ servicios; pero esto no será tan fácil, los organismos rectores deben de diagnosticar, proponer y sugerir las nuevas medidas a tomar; frente a ello propondremos además a la Cámara de Comercio y sus afiliados a gestionar adecuadamente los riesgos que se presente como consecuencia de la incertidumbre post COVID 19. Por ello es necesario redoblar esfuerzos gubernamentales y de parte de la academia proponer alternativas para el retorno a la nueva normalidad.

Cómo objetivo general nos planteamos: determinar la influencia de la gestión de riesgos en la nueva normalidad post COVID 19 y como objetivos específicos: determinar la influencia de la visión holística en la nueva normalidad post COVID 19. Determinar la influencia de la gestión de los riesgos emergentes en la nueva normalidad post COVID $19 \mathrm{y}$ Determinar la influencia de eventos significativos en la nueva normalidad post COVID 19.
Cómo hipótesis general planteamos: la gestión de riesgos influye significativamente en la nueva normalidad post COVID 19 y como hipótesis específicas; lavisiónholísticainfluyesignificativamente en la nueva normalidad post COVID 19, La gestión de los riesgos emergentes influye significativamente en la nueva normalidad post COVID 19 y La gestión de eventos significativos influye significativamente en la nueva normalidad post COVID 19.

\section{MATERIAL Y MÉTODOS}

El trabajo de investigación es el básico. Según Hernández (2017) : “La investigación básica es la que tiene interrogantes más generales y abstractas y aspira a generar conocimiento mediante la formulación de teorías. Sus resultados ayudan a comprender el mundo que nos rodea". Mediante la investigación estudiaremos la influencia de la gestión de riesgos en la nueva normalidad post Covid 19, que a su vez nos servirá para proponer respuestas para superar las incertidumbres que se presentarán.

El diseño de la investigación será de naturaleza no experimental, de corte transversal correlacionalcausal, es no experimental porque solo se observan los fenómenos en su ambiente natural para después analizarlos, es transversal porque se tomarán datos en un punto en el tiempo y correlacional-causal porque para este caso se describen relaciones de causalidad entre la gestión de riesgos y la nueva normalidad post COVID 19.

Las variables son:

$\mathrm{X}=$ Gestión de riesgos

$\mathrm{Y}=$ Nueva normalidad post COVID 19

La población en la presente investigación es de 469 empresarios socios de la Cámara de Comercio de Huancayo. Para nuestra investigación se ha considerado el muestreo hemos utilizado una prueba piloto aplicado a 10 socios de la Cámara de Comercio aleatoriamente y se le pregunto; ¿Usted tiene implementado los protocolos para afrontar los riesgos en su empresa?, el Complejo Hospitalario Universitario de Albacete (2018) manifiesta que de acuerdo a la población y muestra se tiene que utilizar el muestreo. En nuestra investigación se tomará el muestreo estratificado, por la razón de que la Cámara de Comercio agrupa a empresarios de diferentes sectores y que se tomará muestra de cada sector, razón por la cuál será un muestreo estratificado. 
Para el análisis estadístico en la investigación, se tendrá el soporte del software estadístico SPSS versión 25 y de Microsoft Excel. La base de datos se creará en SPSS, a partir de la información recopilada a través de los instrumentos de recolección de datos (cuestionario), y de la matriz tripartita.

\section{RESULTADOS}

A partir de los resultados obtenidos para verificar la influencia de la gestión de riesgos en la nueva normalidad post COVID 19. Para nuestra variable de gestión de riesgos en el direccionamiento estratégico para la identificación de los riesgos son el $42 \%$. La gestión de riegos emergentes como la prevención, documentación y la gestión cuentan con un $37 \%$. En las actividades de seguimiento, control y análisis de riesgos con un $35 \%$ de aplicación.

En cuanto a la nueva normalidad post covid-19, se cuentan con un $39 \%$ el plan para la vigilancia, prevención y control de Covid-19 en el trabajo. Con un $39 \%$ cuentan con equipos de protección personal anti Covid-19 y protocolos para la atención y los procesos en el trabajo. En la salud ocupacional un 36\% cuentan con los elementos del lugar de trabajo para evaluar los peligros y los riesgos.

\section{DISCUSIÓN}

Para confrontar los resultados obtenidos con otras investigaciones es necesario identificar el marco teórico, técnicas aplicadas en la investigación y la aplicación de los instrumentos y así determinar la influencia de la gestión de riesgos en la nueva normalidad post COVID 19.

La Gestión de riesgos, que es un proceso de planificación, organización, dirección y control de los recursos humanos y materiales de una organización, con el fin de reducir al mínimo o aprovechar los riesgos e incertidumbres de la organización. Las incertidumbres representan riesgos y oportunidades con el potencial de destruir o crear valor. La gestión de riesgos de la empresa permite a los administradores hacer frente eficazmente a las incertidumbres, así como a los riesgos y oportunidades asociados con ellos, con el fin de mejorar la capacidad de generar valor. El valor se maximiza cuando la organización establece estrategias y objetivos para lograr el equilibrio ideal entre los objetivos de crecimiento, rentabilidad de la inversión y los riesgos asociados con ellos, y para explorar sus recursos con eficacia y eficiencia en la consecución de objetivos de la organización (Oliveira, 2020).

El Instituto para una Cultura de Seguridad Industrial [ICSI] (2020), considera que se debe articular a los grupos de intercambio y de trabajo, para gestionar los riesgos, para el cual sugiere crear grupos de intercambio dedicado a reexaminar las prácticas de gestión de los riesgos a la luz de las enseñanzas de la crisis del Covid 19. Estos intercambios realizados ya han hecho surgir temas que, a pesar de ser conocidos, vuelven a estar en un primer plano, como la resiliencia de las organizaciones para tener un buen nivel de seguridad, el estilo de liderazgo en seguridad que se necesita para hacer frente a una incertidumbre como la que atravesamos, la adaptación o no de los modelos de seguridad tradicionales ahora en base a normas de calidad que sugieren la reducción del impacto de los riesgos.

La pandemia de COVID 19 está madurando, pero las incertidumbres continúan multiplicándose tanto para los individuos como para los responsables políticos. ¿Debo volver al trabajo? ¿Debo visitar a un pariente? ¿Qué empresas deberían reabrir? ¿Qué pasa con las escuelas y universidades? Por tanto, la incertidumbre y de cómo la manejamos tanto a nivel personal como político cuando la acción urgente es esencial depende de la toma de decisiones. La ciencia a veces se representa como una búsqueda metódica y minuciosa de la verdad, y una buena formulación de políticas como la traducción de esas verdades basadas en la evidencia a la acción. En tiempos anteriores a la pandemia, estos supuestos a veces (aunque no siempre) se sostuvieron. Pero la complejidad de la ciencia y la formulación de políticas en el contexto de la incertidumbre se han puesto de manifiesto en esta pandemia.

La enfermedad es nueva probablemente, algunos hallazgos de investigaciones recientes pueden tener el estado de los hechos, pero en general, la evidencia basada en la efectividad de las intervenciones, tanto preventivas como terapéuticas, sigue siendo irregular y limitada. Se cuestiona la medida en que los resultados de la investigación de otras enfermedades (e incluso otros coronavirus) se pueden extrapolar a COVID-19, en tanto, la incertidumbre vive con nosotros.

\section{CONCLUSIONES}

Al concluir la investigación se puede afirmar a un nivel de significancia del 0,05 que la gestión de riesgos 
influye significativamente en la nueva normalidad post COVID 19. Lo cual significa que la gestión de riesgos influye hasta un $58,5 \%$ en la nueva normalidad post COVID 19.

Se puede afirmar a un nivel de significancia de la visión holística influye significativamente en la nueva normalidad post COVID 19. Lo cual significa la visión holística influye hasta un 52,4\% en la nueva normalidad post COVID 19.

Se puede afirmar a un nivel de significancia la gestión de los riesgos emergentes influye significativamente en la nueva normalidad post COVID 19. Lo cual significa que la gestión de los riesgos emergentes influye hasta un $48,3 \%$ en la nueva normalidad post COVID 19.

Se puede afirmar a un nivel de significancia de la gestión de eventos significativos influye significativamente en la nueva normalidad post COVID 19. Lo cual significa que la gestión de eventos significativos influye hasta un $55.8 \%$ en la nueva normalidad post COVID 19.

\section{REFERENCIAS BIBLIOGRÁFICAS}

1. Complejo Hospitalario Universitario de Albacete. (2018). Muestreo. Complejo Hospitalario Universitario de Albacete. https://www.chospab.es/ calidad/archivos/Metodos/Muestreo.pdf

2. Hernández, R. (2017). Fundamentos de Investigación. Ciudad de México: McGraw Education.

3. Instituto para una Cultura de Seguridad Industrial. (2020). La seguridad del mañana. Instituto para una Cultura de Seguridad Industrial. https://www.icsi-eu. org/es/revista/gestion-riesgos-covid-programa

4. Oliveira, W. (2020). ¿Qué es la gestión de riesgos? Propósitos y concepto. HEFLO. https://www.heflo. $\mathrm{com} / \mathrm{es} / \mathrm{blog} /$ gestion-de-riesgos/que-es-gestion-deriesgos/

5. Pérez, Y. (2021). Claves para reforzar la gestión de riesgos tras COVID-19. KPMG Tendencias. https://www.tendencias.kpmg.es/2020/04/clavesreforzar-gestion-riesgos-covid-19/

Recibido: 04/06/2021 Aceptado: 17/10/2021 